\title{
Correcciones de una imagen satelital ASTER para estimar parámetros vegetacionales en la cuenca del río Mirta, Aisén
}

\author{
ASTER satellite image corrections to estimate vegetational parameters \\ on the Mirta river basin, Aisén
}

\author{
Marco Antonio Peña Araya \\ Consultora Teledetección, Manuel Barrios 5655, Las Condes, Santiago, Chile, Tel.: 56-2-7245725, \\ mpena@teledeteccion.cl
}

\begin{abstract}
SUMMARY
This work evaluated the effect of different correction procedures of an ASTER satellite image on the estimation of Vegetation Cover Fraction (VCF) and Normalized Difference Vegetation Index (NDVI) on the Mirta river basin, located in Cisnes county, Aisén Region, Chile. Specifically, atmospheric, topographic and sub-pixel rectification procedures were applied on the image. That allowed to estimate both vegetational parameters on uncorrected data and on those subjected to different correction levels, and thus to establish frequency and agreement comparisons among their classes. After subjecting the image to the mentioned corrections, estimation of VCF and NDVI yielded values markedly higher than those estimated from the raw image. Classes of the VCF image that incorporated a sub-pixel rectification exhibited the lowest agreement in relation to their equivalents estimated from raw image, while NDVI estimated from the atmospherically rectified image presented a larger proportion of values near to the greenness saturation, which were not rendered when this parameter was estimated from the raw image data. The relation of VCF and NDVI with the main vegetation covers of the basin allowed to observe that both parameters exhibit their highest values on dense forest formations, while their lowest values are present on scrub formations or forest formations mixed with young trees.
\end{abstract}

Key words: vegetation cover fraction, normalized difference vegetation index, radiometric corrections, spectral mixture analysis, Aisén.

\section{RESUMEN}

Este trabajo evaluó el efecto de diferentes procedimientos de corrección de una imagen satelital ASTER sobre la estimación de la fracción de cobertura vegetal (VCF) y el índice de vegetación de diferencia normalizada (NDVI) en la cuenca del río Mirta, localizada en la comuna de Cisnes, Región de Aisén, Chile. Específicamente, fueron aplicados procedimientos de corrección atmosférica, topográfica y de subpíxel sobre la imagen. Ello permitió estimar ambos parámetros vegetacionales sobre los datos sin corregir y sobre aquellos sometidos a los diferentes niveles de corrección, para luego establecer comparaciones de frecuencia y concordancia entre sus valores discretizados en clases. Luego de someter la imagen a las correcciones señaladas, la estimación de VCF y NDVI arrojó valores marcadamente más elevados que los estimados desde la imagen cruda. Las clases de la imagen de VCF que incorporó una corrección de subpíxel exhibieron la concordancia más baja en relación con sus equivalentes estimadas desde la imagen cruda, mientras que el NDVI estimado desde la imagen rectificada atmosféricamente presentó una gran proporción de valores cercanos a la saturación de verdor, los que no fueron arrojados cuando este parámetro fue estimado desde los datos crudos de la imagen. La relación de la VCF y el NDVI con las principales coberturas vegetales de la cuenca permitió observar que ambos parámetros presentan sus mayores valores en formaciones de bosque denso, en tanto que sus menores valores se aprecian en formaciones de matorral o formaciones de bosque mezclado con renovales.

Palabras clave: fracción de cobertura vegetal, índice de vegetación de diferencia normalizada, correcciones radiométricas, análisis de mezcla espectral, Aisén.

\section{INTRODUCCIÓN}

Las imágenes satelitales registran el comportamiento de la superficie terrestre a través de diferentes regiones del espectro electromagnético (ello depende de la resolución espectral de la imagen, es decir, del número de bandas o rangos de longitudes de onda discriminados en ésta), proporcionando una gran cantidad de datos espacialmente contiguos entre sí (éstos se ordenan en una grilla de celdas cuadradas o píxeles, que almacenan un valor de energía electromagnética en una unidad de medida llamada radiancia) y distribuidos a lo largo de extensas áreas geográficas (las escenas completas de imágenes de baja y moderada resolución espacial cubren miles de 
kilómetros cuadrados). Estas propiedades les confieren la capacidad de detectar, reconocer e identificar coberturas de suelo, así como medir numerosas propiedades biofísicas y bioquímicas asociadas a ellas, ofreciendo ventajas en comparación con métodos in situ, que muchas veces requieren de mediciones en terreno que pueden resultar prohibitivas a amplias escalas, debido a limitantes de accesibilidad, tiempo y recursos (Lillesand et al. 2004, Aronoff 2005, Eastman 2006).

Debido a lo anterior, en numerosos países europeos y de Norteamerica las imágenes satelitales han contribuido a caracterizar y monitorear el recurso bosque a través del cálculo de medidas relacionadas a abundancia y estado de salud vegetal (Iverson et al. 1989, Purevdorj et al. 1998). No obstante, previo a la realización de estos cálculos los valores de radiancia de las diferentes bandas que componen a una imagen requieren necesariamente de la aplicación de una serie de procedimientos de corrección, en especial si se desea estimar alguna propiedad con un sentido físico preciso, como es el caso de la fracción de cobertura vegetal (VCF, vegetation cover fraction), o relacionar cuantitativamente una estimación con la fisiología de la vegetación, como ocurre con el índice de vegetación de diferencia normalizada (NDVI, normalized difference vegetation index). La VCF corresponde al porcentaje de área al interior de cada píxel de la imagen que es ocupada por vegetación y cuyo valor puede ser expresado en una determinada unidad de superficie de acuerdo a la dimensión del píxel. El NDVI corresponde a una medida relativa acerca del verdor o vigor de la vegetación contenida en los píxeles de la imagen. Ambos parámetros vegetacionales han sido profusamente correlacionados con variables como abundancia, sanidad, biomasa e índice de área foliar (Purevdorj et al. 1998, Zheng et al. 2004, Freitas et al. 2005, Labrecque et al. 2006).

Si bien los valores de radiancia codificados en una imagen deberían representar sólo la energía reflejada o emitida por la superficie terrestre, ellos siempre estarán alterados por la dispersión y emisión de energía que producen los aerosoles atmosféricos y por la iluminación diferenciada de la superficie terrestre, producto de la interacción de la radiación solar con el relieve y sus elementos. Estos factores modifican especialmente la radiancia de las bandas visible e infrarrojo cercano de una imagen, de las que precisamente depende la estimación de cualquier parámetro vegetacional, pues abarcan las longitudes de onda en que la vegetación posee un comportamiento espectral distintivo (i.e. patrón espectral) que permite su detección (Riaño et al. 2003, Lillesand et al. 2004, Aronoff 2005). Por esta razón, antes de estimar parámetros como la VCF o el NDVI es necesario corregir la imagen bajo el supuesto que sus datos fueron adquiridos en condiciones de observación perfectas e invariantes, en particular si se trata de áreas especialmente sujetas a los efectos detallados a continuación.
Efecto atmosférico. La energía radiada por la superficie terrestre debe recorrer una cierta distancia a través de la atmósfera antes de ser detectada por un sensor, la cual es conocida como longitud de ruta (path length). Durante este viaje, el flujo radiante es esparcido y absorbido por los aerosoles atmosféricos, lo que modifica la radiancia que finalmente registran los detectores del sensor desde su objetivo. Específicamente, el proceso de esparcimiento redirecciona energía que se añade como radiancia de ruta extraña (extraneous path radiance) a la señal medida por el sensor, mientras que el proceso de absorción atenúa la señal de energía que éste recibe (Lillesand et al. 2004, Aronoff 2005). En la imagen, el esparcimiento Rayleigh suele ser el más evidente, y es ocasionado por pequeños aerosoles atmosféricos que redireccionan energía de onda corta que se añade a la radiancia de los píxeles (i.e. efecto de niebla), reduciendo la visualización de detalles (debido a que la imagen pierde contraste y brillo, es decir, disminuye su detalle radiométrico) e induciendo a una estimación incorrecta de algún parámetro vegetacional (debido a que los valores de radiancia se encuentran sobreestimados) (Lillesand et al. 2004, Aronoff 2005). Este efecto puede ser minimizado mediante la aplicación de un modelo de corrección atmosférica sobre las bandas de la imagen.

Efecto topográfico. La superficie terrestre es irregular y la radiación solar sólo en un momento específico incide sobre ella de forma completamente vertical (i.e. cenit solar), por lo que la radiancia medida por un sensor suele estar sujeta a la iluminación diferenciada del relieve que resulta de la interacción de su topografía (i.e. pendiente y exposición) con la posición solar (i.e. elevación y azimut solar) (Eastman 2006). Dado que las rutas orbitales de los satélites a menudo están diseñadas para cubrir áreas de interés durante las primeras horas de la mañana o alrededor de mediodía, el ángulo de elevación solar al momento de adquisición de una imagen frecuentemente es oblicuo. Por otra parte, la latitud y la estación del año juegan un rol determinante en la posición solar presente a la hora de paso de un satélite por un área dada. De esta manera, en relieves montañosos la radiancia medida por un sensor desde una cobertura de suelo situada en una ladera de exposición umbría (i.e. oculta al sol) puede ser considerablemente menor que aquella medida desde la misma cobertura de suelo situada en una ladera de exposición solana (i.e. expuesta al sol), dificultando el cálculo fidedigno de algún parámetro vegetacional, pues el comportamiento espectral de la vegetación se ve alterado por la presencia de sombras (Riaño et al. 2003, Eastman 2006). Este efecto puede ser minimizado mediante la aplicación de un modelo de corrección topográfica sobre las bandas de la imagen.

Efecto del dosel vegetal. Un árbol, al interactuar con la radiación solar, proyecta una sombra cuyo largo dependerá de su altura y del ángulo de elevación solar. En un bosque 
compuesto por individuos de diferentes alturas y sometidos a un ángulo de incidencia solar (i.e. aquel formado por el ángulo de elevación solar y la normal a la pendiente) oblicuo, se producen numerosas sombras que se proyectan sobre la superficie del dosel superior. En la imagen, este efecto limita la detección de vegetación, tanto si se trata de procedimientos de píxel completo (e.g. NDVI) como de subpíxel (e.g. VCF), pues la vegetación afectada por sombras diferirá espectralmente de la vegetación iluminada, conllevando a subestimar su real presencia en el píxel. Dado que usualmente la longitud de este tipo de sombras es inferior a la resolución espacial de un modelo de corrección topográfica convencional (ello dependerá del modelo digital de elevación (DEM) empleado), sólo mediante la aplicación de técnicas de descomposición espectral del píxel (pixel spectral unmixing) es posible identificar la proporción de vegetación ensombrecida y estimar su presencia al interior de él (Asner et al. 2003, Aronoff 2005).

El objetivo de este trabajo es evaluar el efecto que producen los procedimientos de corrección mencionados sobre la estimación de la VCF y el NDVI. Para ello se ha empleado una imagen satelital ASTER (Advanced Spaceborne Thermal Emission and Reflection Radiometer) correspondiente a la cuenca del río Mirta, localizada en la comuna de Cisnes, en el extremo noreste de la Región de Aisén, Chile. Esta área posee características favorables para cumplir el objetivo planteado, pues se inserta en un clima húmedo con abundantes lluvias, en que más del $50 \%$ de los días del año se encuentra nublado o parcialmente nublado y sólo el 3\% de ellos se encuentra totalmente despejado (Donoso 1981), lo que debería propiciar un alto contenido de humedad en la atmósfera y con ello acentuar el efecto de niebla en la imagen. La cuenca se localiza aproximadamente a $43^{\circ} 52^{\prime}$ ' de latitud Sur y está limitada por montañas que superan los $1.500 \mathrm{~m}$ de elevación, con laderas que exceden el $30 \%$ de pendiente (CONAF et al. 1999), lo que debería agudizar la iluminación diferenciada del relieve y con ello acentuar la presencia de laderas ensombrecidas en la imagen. Además, los antecedentes proporcionados por observaciones realizadas en terreno e información auxiliar (CONAF et al. 1999, CONAF 2000) permiten constatar que gran parte de las laderas del área de estudio presentan densas y bien desarrolladas formaciones de bosque siempreverde (en el valle han sido interrumpidas por praderas y matorrales, encontrándose más ralas como resultado de una mayor intervención antrópica), caracterizadas por una composición de variadas especies multietáneas que producen intensas sombras (Donoso 1981), lo cual debería afectar la iluminación de la superficie del dosel vegetal superior representado en la imagen. Cada uno de los factores enunciados limita la detección de vegetación en la imagen y por lo tanto la estimación de la VCF y el NDVI.

\section{MÉTODOS}

Preprocesamiento de la imagen. Los parámetros de VCF y NDVI fueron estimados a partir del procesamiento de una imagen satelital ASTER, adquirida el 17 de febrero de 2002, a las 11:55 a.m. hora local. Esta imagen se caracteriza por poseer una mayor resolución espectral que las tradicionales imágenes multiespectrales, lo que le otorga una mejor capacidad para discriminar coberturas de suelo. Específicamente, distribuye 14 bandas en el espectro electromagnético: tres en las dimensiones visible e infrarrojo cercano (VNIR, visible and near infrared); seis en la dimensión infrarrojo de onda corta (SWIR, shortwave infrared), y cinco en la dimensión infrarrojo térmico (TIR, thermal infrared). Con el sentido de no perder nivel de detalle espacial, las bandas fueron procesadas con una resolución espacial de $15 \mathrm{~m}$, que corresponde al tamaño de píxel de las bandas VNIR (dado que las bandas SWIR y TIR poseen una resolución espacial de 30 y $90 \mathrm{~m}$, respectivamente, su resolución espacial puede ser ajustada a la de las bandas VNIR sin degradar su detalle espacial).

La imagen empleada se distribuye georreferenciada con coordenadas geográficas UTM (Universal Transversal de Mercator) (nivel de datos 1B), de modo que sólo fue necesario ortorrectificarla para otorgarle una corrección geométrica más completa. Luego, fue definida una subescena correspondiente al área de estudio, la cual fue sometida a un procedimiento de corrección atmosférica mediante el empleo del modelo FLAASH (fast line-of -sight atmospheric analysis of spectral hypercubes), que permitió reducir la radiancia añadida a los píxeles de cada banda por el efecto de niebla. Posteriormente, fue sujeta a un procedimiento de corrección topográfica mediante el empleo de un modelo de Reflexión Lambertiana, que con la asistencia de un DEM de $50 \mathrm{~m}$ de resolución espacial permitió normalizar las condiciones de iluminación del relieve y rectificar los valores de radiancia de aquellos píxeles afectados por sombra. Dado que el DEM y la imagen ASTER poseen resoluciones espaciales moderadas (la imagen comprende niveles de detalle de entre 15 y 90 m), la corrección topográfica es factible sin generalizar significativamente la sinuosidad del relieve representado en esta última.

Obtención del NDVI. El NDVI fue estimado mediante la siguiente combinación aritmética de bandas [1]:

$$
\mathrm{NDVI}=\mathrm{NIR}-\mathrm{R} / \mathrm{NIR}+\mathrm{R}
$$

Donde:

NIR: banda del infrarrojo cercano.

R: banda del rojo.

Los valores de la imagen de salida proporcionan una medida relativa de verdor de la vegetación a partir del contraste espectral que se produce entre ambas bandas (en 
$\mathrm{R}$ la clorofila refleja poca radiación y en NIR las hojas reflejan mucha radiación), en donde los valores inferiores o equivalentes a 0 corresponden a suelo desnudo y aquellos cercanos a 1 representan a una superficie saturada de vegetación verde (i.e. no existe suelo desnudo o vegetación senescente al interior del píxel) (Eastman 2006).

Dado que este índice es estimado mediante una división de bandas, la imagen no requiere previamente de una corrección topográfica, pues aunque en cada banda los valores de radiancia de un mismo tipo de cobertura de suelo varíen según su exposición, mantendrán una idéntica razón al ser divididas entre sí (Lillesand et al. 2004). Asimismo, al ser un parámetro de píxel completo (i.e. el píxel adopta un único valor a pesar de estar compuesto por distintos tipos de elementos) no es posible identificar la proporción de vegetación en sombra contenida al interior de éste, de tal modo que este tipo de corrección tampoco puede ser efectuada.

De esta manera, fueron generadas dos imágenes de NDVI: NDVI0, resultante de la imagen cruda (i.e. $\sin$ rectificar) y NDVI1, resultante de la imagen sometida a corrección atmosférica.

Obtención de la VCF. La VCF fue estimada mediante una técnica de análisis de mezcla espectral (spectral mixture analysis), que proporciona una estimación de la proporción de área aproximada que ocupa una cobertura de interés al interior de cada píxel de la imagen. Específicamente, fue empleado un algoritmo de descomposición espectral lineal (linear spectral unmixing), el cual asume que la radiancia de un píxel es el resultado de la combinación lineal de un limitado conjunto de patrones espectrales puros (i.e. endmembers) correspondientes a los variados tipos de coberturas de suelo representados en la imagen (Purevdorj et al. 1998, Brown 2001, Lillesand et al. 2004).

Para ello, este algoritmo extrae desde áreas de entrenamiento (i.e. polígonos que el analista delimita sobre un rasgo de interés en la imagen) el comportamiento espectral de una cobertura de suelo, para luego identificarla al interior de un píxel y estimar, a partir de su contribución en la radiancia codificada, la participación porcentual de área que tiene al interior de él (Purevdorj et al. 1998, Lillesand et al. 2004, Aronoff 2005).

Es importante que las áreas de entrenamiento delimiten sólo la cobertura de suelo de interés, de manera que el comportamiento espectral extraído sea "puro", permitiendo la estimación correcta de la fracción de dicha cobertura en el píxel. Debido a lo anterior, las áreas de entrenamiento para estimar la VCF del área de estudio fueron delimitadas sobre superficies de muy alto NDVI $(\approx 1)$. Además, la técnica de descomposición espectral permitió estimar el porcentaje de suelo desnudo, agua y hielo presente en los píxeles de la imagen, lo que permitió enmascarar y sustraer de los análisis aquellas superficies desprovistas de vegetación (i.e. cuerpos de agua, glaciares, rocas, etc.).
Adicionalmente, a través de observaciones realizadas en terreno fue posible identificar superficies cubiertas de vegetación que en la imagen se visualizan ensombrecidas. Sobre ellas fueron realizadas áreas de entrenamiento, para luego aplicar el algoritmo de descomposición espectral, que permitió estimar el porcentaje de área de cada píxel de la imagen correspondiente a vegetación ensombrecida. Estos valores fueron sumados a la imagen de VCF corregida atmosférica y topográficamente con el propósito de obtener una estimación más correcta de este parámetro.

A partir de los procedimientos de corrección efectuados fueron generadas cuatro imágenes de VCF: VCF0 = resultante de la imagen cruda, VCF1 = resultante de la imagen sometida a corrección atmosférica, $\mathrm{VCF} 2$ = resultante de la imagen rectificada atmosférica y topográficamente y VCF3 = resultante de la imagen sometida a las rectificaciones precedentes y con la vegetación ensombrecida incorporada.

Con el propósito de calcular la superficie total afectada por sombras topográficas en el área de estudio, fueron elaboradas imágenes de pendiente y exposición del relieve a partir de un DEM con resolución espacial de $50 \mathrm{~m}$. Posteriormente, desde el archivo de cabecera de la imagen fueron tomados los datos de azimut y elevación solar al momento de paso del satélite Terra (plataforma espacial que transporta al sensor ASTER). El primer antecedente permitió definir la exposición del relieve y el segundo definir el umbral de pendiente a partir del cual las laderas no reciben radiación solar directa.

Comparación de imágenes. Para evaluar los resultados obtenidos en el caso del NDVI y de la VCF, los valores de cada una de las imágenes rectificadas fueron comparados estadística y espacialmente con los correspondientes a las imágenes crudas. Con el sentido de facilitar tales comparaciones los valores de NDVI y VCF fueron discretizados en las siguientes clases: (1) inferior a $25 \%$, (2) entre 25 y $50 \%$, (3) entre 50 y $75 \%$ y (4) superior a $75 \%$.

Lo anterior permitió comparar la frecuencia absoluta y relativa de las clases crudas y rectificadas de las imágenes de NDVI y VCF separadamente, así como medir el grado de variación que experimentó cada clase de la imagen cruda en relación con su equivalente en las imágenes rectificadas, para lo cual fue aplicado el índice de concordancia de Kappa (KIA, Kappa Index of Agreement). Este índice mide la similitud entre clases equivalentes de dos imágenes comparadas (i.e. comparación clase por clase), donde una de ellas es considerada como de referencia y la otra como de ajuste. El valor 1 indica que la clase de la imagen de ajuste se superpone perfectamente a su equivalente en la imagen de referencia, mientras que el valor 0 indica que la clase de la imagen de ajuste no se superpone a su equivalente en la imagen de referencia (Pontius 2000, Lillesand et al. 2004). Este índice fue obtenido automáticamente mediante el empleo de un sistema de información geográfica, considerando la imagen cruda como de ajuste 
y las imágenes rectificadas como de referencia, tanto para el caso del NDVI como para el de VCF.

Empleo de información auxiliar. Dado que este trabajo no contó con mediciones in situ de variables equivalentes a la VCF y el NDVI, fue imposible evaluar el grado de precisión de los valores observados remotamente en relación con aquellos observados en terreno (ground truth), ya sea mediante la confección de una matriz de error o el cálculo del índice de Kappa, pues ambos métodos exigen que los datos comparados se refieran a la misma variable.

Sin embargo, los valores de ambos parámetros vegetacionales fueron contrastados y analizados en relación con las principales clases de cobertura vegetal de la cuenca, que fueron tomadas del proyecto Catastro de Bosque Nativo correspondiente a la Región de Aisén (CONAF et al. 1999). De acuerdo con la información proporcionada por este proyecto, en la cuenca se presentan siete grandes clases de cobertura vegetal, diferenciadas según tipo de formación y densidad. Estas clases fueron transformadas de su formato vectorial original a formato raster, con la finalidad de superponerlas a las imágenes de ambos parámetros vegetacionales y calcular los valores promedio que éstos presentan en cada una de ellas. La descripción de las clases fue apoyada mediante información levantada en terreno.

Este procedimiento permitió otorgar una mayor interpretación a los valores de VCF y NDVI del área de estudio, a la vez de proporcionar una noción acerca de la relación que ellos presentan según el tipo de formación y la densidad de cada clase de cobertura vegetal.

\section{RESULTADOS}

Los antecedentes del archivo de cabecera de la imagen procesada indican que durante el paso del satélite Terra por el área de estudio, la posición solar registró un azimut de $50,64^{\circ}$ y una elevación de $47,9^{\circ}$, de modo que al momento de adquisición de la imagen todos aquellos relieves orientados al sur y oeste, y con pendientes superiores a $47,9^{\circ}$, se encontraban ocultos al sol, limitando la detección de rasgos geográficos y coberturas de suelo por parte del sensor. Las superficies de exposición umbría abarcan el 18\% del área de estudio y las superficies no vegetadas que fueron enmascaradas y sustraídas de los análisis corresponden al $16 \%$ del área de estudio (cuadro 1).

Cuadro 1. Área según tipo de superficie. Area according to surface type.

\begin{tabular}{lcc}
\hline Tipo de superficie & ha & $\%$ \\
\hline Roca, hielo y cuerpos de agua & 5.618 & 16 \\
Exposición umbría & 6.198 & 18 \\
Exposición solana & 23.119 & 66 \\
Total área de estudio & 34.935 & 100 \\
\hline
\end{tabular}

La figura 1 presenta dos imágenes ASTER compuestas en falso color del área de estudio; la inferior cruda y la superior sometida a rectificaciones de tipo atmosférica y topográfica. En esta última, se han normalizado las condiciones de iluminación de la superficie, reduciendo la cantidad e intensidad de las sombras, de manera que el relieve luce plano (algunas superficies de roca y hielo mantienen sombras, ya que al no presentar interés para el presente trabajo su corrección topográfica no fue acuciosa). Los antecedentes del archivo de cabecera de la imagen
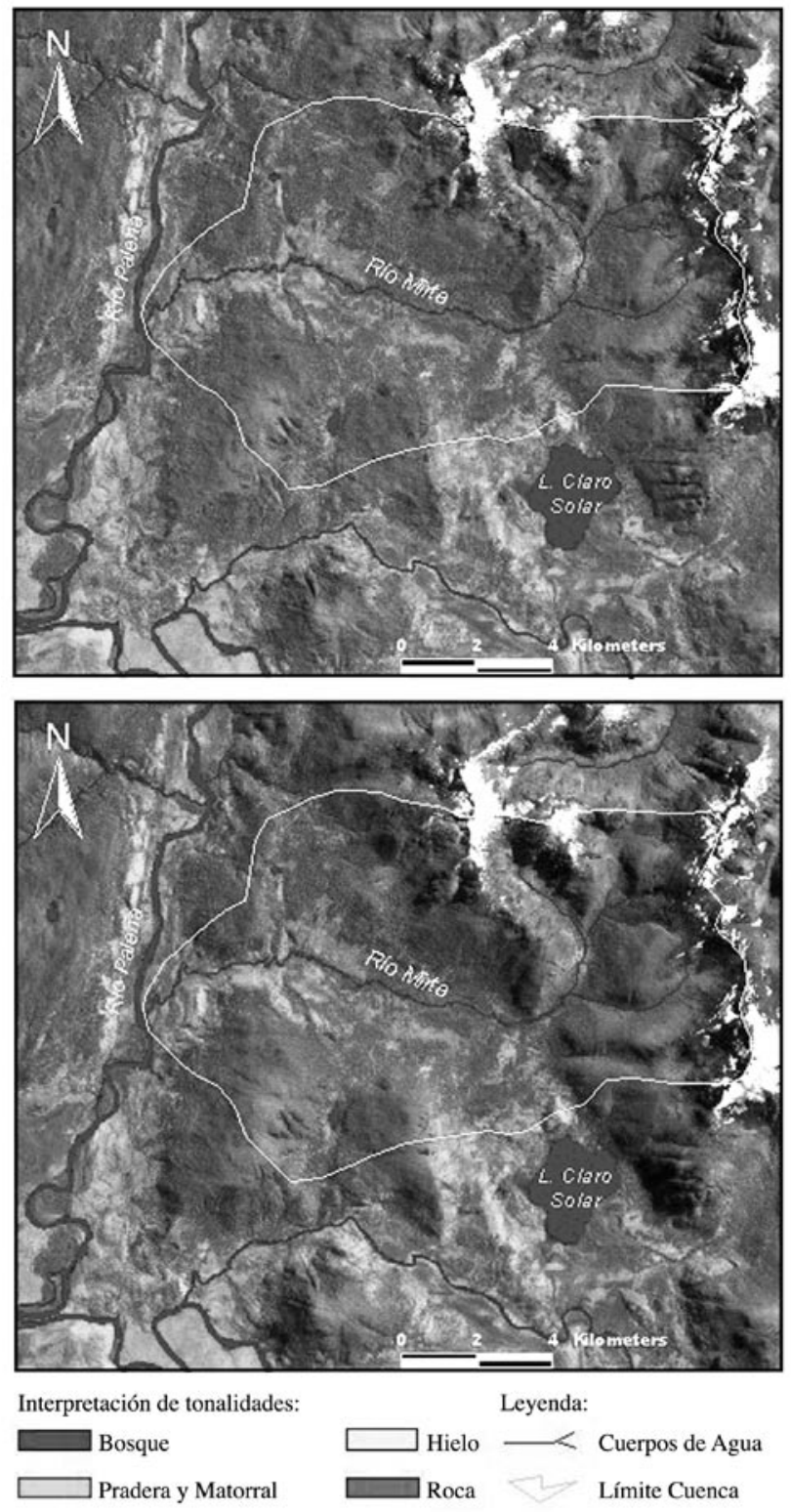

Figura 1. Ambas imágenes compuestas corresponden a la cuenca del río Mirta y sus alrededores. La inferior no ha sido rectificada atmosférica y topográficamente, mientras que la superior ha sido sometida a ambas rectificaciones.

Both composite images correspond to the Mirta river basin and its surroundings. The inferior image has not been atmospherically or topographically corrected, while the superior image has been subjected to both corrections. 
Cuadro 2. Frecuencia de clases según imagen de VCF.

Frequency classes according to VCF image.

\begin{tabular}{|c|c|c|c|c|c|c|c|c|c|}
\hline \multirow{2}{*}{ Clase } & \multirow{2}{*}{$\begin{array}{c}\text { Rango de } \\
\text { valores }(\%)\end{array}$} & \multicolumn{2}{|c|}{ VCF0 } & \multicolumn{2}{|c|}{ VCF1 } & \multicolumn{2}{|c|}{ VCF2 } & \multicolumn{2}{|c|}{ VCF3 } \\
\hline & & $\%$ & ha & $\%$ & ha & $\%$ & ha & $\%$ & ha \\
\hline 1 & 0 a 25 & 10 & 2.864 & 12 & 3.480 & 7 & 2.081 & 3 & 933 \\
\hline 2 & 25 a 50 & 43 & 12.678 & 48 & 13.949 & 47 & 13.665 & 24 & 6.940 \\
\hline 3 & 50 a 75 & 38 & 11.157 & 33 & 9.684 & 38 & 11.129 & 34 & 10.116 \\
\hline 4 & 75 a 100 & 9 & 2.618 & 7 & 2.204 & 8 & 2.442 & 39 & 11.328 \\
\hline Total & - & 100 & 29.317 & 100 & 29.317 & 100 & 29.317 & 100 & 29.317 \\
\hline
\end{tabular}

señalan que al momento de su adquisición la nubosidad era cercana a $0 \%$. Esta nitidez atmosférica contribuyó a reducir el efecto de niebla en la imagen, por cuanto el vapor de agua es uno de los principales aerosoles que redirecciona energía electromagnética. De esta manera, luego de la aplicación de la corrección atmosférica no se evidencia a simple vista un mejoramiento del detalle radiométrico de la imagen (i.e. ajuste del contraste y brillo). Sin embargo, como se comprobará más adelante, los valores de radiancia de sus píxeles sí variaron luego de ser sometidos a este procedimiento de corrección. Ello es confirmado por la variación que presentaron los valores de VCF y NDVI una vez aplicada esta corrección.

Caso de la VCF. La media de valores de VCF0, VCF1 y VCF2 corresponde a 49,10, 46,25 y 49,37\%, respectivamente. Estos resultados denotan que una vez aplicados los procedimientos de corrección atmosférica y topográfica sobre la imagen, los valores de este parámetro varían poco. Sin embargo, una vez aplicada la corrección de subpíxel (VCF3) la media de valores aumenta a 66,93\%.

El cuadro 2 permite comparar las frecuencias relativas y absolutas de las clases de cada imagen de VCF. En VCF0, VCF1 y VCF2 la mayor frecuencia de valores se encuentra en la clase 2, seguida de la clase 3 , mientras que en VCF3 la mayor frecuencia de valores se encuentra en la clase 4, seguida de la clase 3. Al comparar VCF0 y VCF1, se observa que en esta última las clases 1 y 2 han aumentado su frecuencia, mientras que las clases 3 y 4 han disminuido su frecuencia. En VCF2 destaca la disminución de la frecuencia de la clase 1 en comparación con VCF0 y VCF1. Por último, al comparar VCF3 con las imágenes restantes, se observa una disminución de las clases 1 y 2 , y un aumento ostensible de la clase 4, que es más de cuatro veces superior que las correspondientes a las imágenes restantes.

Los KIAs presentados en el cuadro 3 destacan una buena concordancia entre las clases de VCF0 y VCF1, particularmente en lo que respecta a la comparación de las clases 1 de ambas imágenes. Entre VCF0 y VCF2 la concordancia de las clases 1 ha bajado notoriamente en relación con la anterior comparación, mientras que en las clases restantes los KIAs han variado muy poco. Finalmente, la comparación de clases entre VCF0 y VCF3 presenta una
Cuadro 3. KIAs obtenidos de la comparación entre clases de VCF.

KIAs retrived from the comparison between VCF classes.

\begin{tabular}{cccc}
\hline \multirow{2}{*}{$\begin{array}{c}\text { Clases } \\
\text { Comparadas }\end{array}$} & \multicolumn{3}{c}{ KIAs entre imágenes de VCF } \\
\cline { 2 - 4 } & VCF0 vs VCF1 & VCF0 vs VCF2 & VCF0 vs VCF3 \\
\hline 1 vs 1 & 0,82 & 0,53 & 0,24 \\
2 vs 2 & 0,70 & 0,67 & 0,22 \\
3 vs 3 & 0,54 & 0,60 & 0,16 \\
4 vs 4 & 0,57 & 0,60 & 0,81 \\
\hline
\end{tabular}

concordancia muy baja, a excepción de la comparación de clases 4, la cual muestra un alto KIA (ello se debe a que los píxeles que presentan la clase 4 en $\mathrm{VCF} 0$ aún se mantienen en VCF3).

La figura 2 muestra la distribución espacial de las clases de cada imagen de VCF, contribuyendo a explicar los KIAs obtenidos de sus comparaciones. Al respecto, la buena concordancia que exhiben las clases de VCF0 y VCF1 se relaciona a la similar distribución espacial que ellas presentan en ambas imágenes, en las que la clase 1 se localiza en laderas de exposición umbría, la clase 2 en laderas de exposición umbría y fondo de valle, la clase 3 en laderas de exposición solana y fondo de valle, y la clase 4 preferentemente en laderas de exposición solana. La disminución del KIA entre las clases 1 de VCF0 y VCF2 en comparación con VCF0 y VCF1 se vincula a la reducción de dicha clase en VCF2, mientras que la notoria variación espacial de las clases de VCF3 en comparación con VCF0 explican los bajos KIAs obtenidos en este caso, observándose un claro aumento de la clase 4, particularmente en laderas, así como una reducción de la clase 1 , restringida casi exclusivamente al límite vegetacional.

Caso del NDVI. La media de valores de NDVI0 corresponde a 44,85\%. Una vez aplicada la corrección atmosférica (NDVI1) la media de valores aumenta a 75,73\%. El cuadro 4 revela que mientras NDVI1 presenta la mayor concentración de valores en las clases 4 y 3 , NDVI0 presenta la mayor frecuencia de valores en las clases 3 y 2 , en tanto que en la clase 4 no presenta valores. 


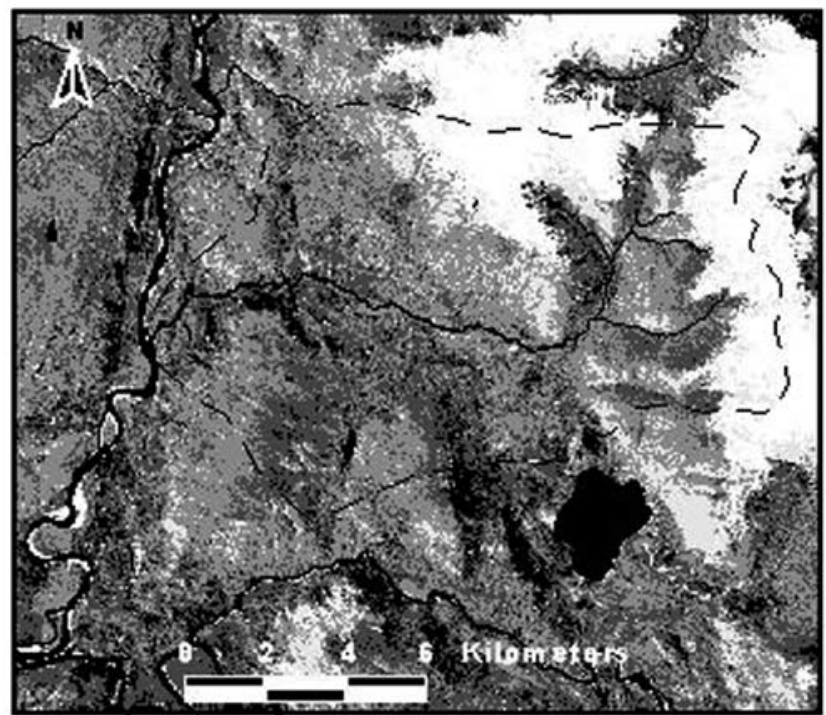

FCV0

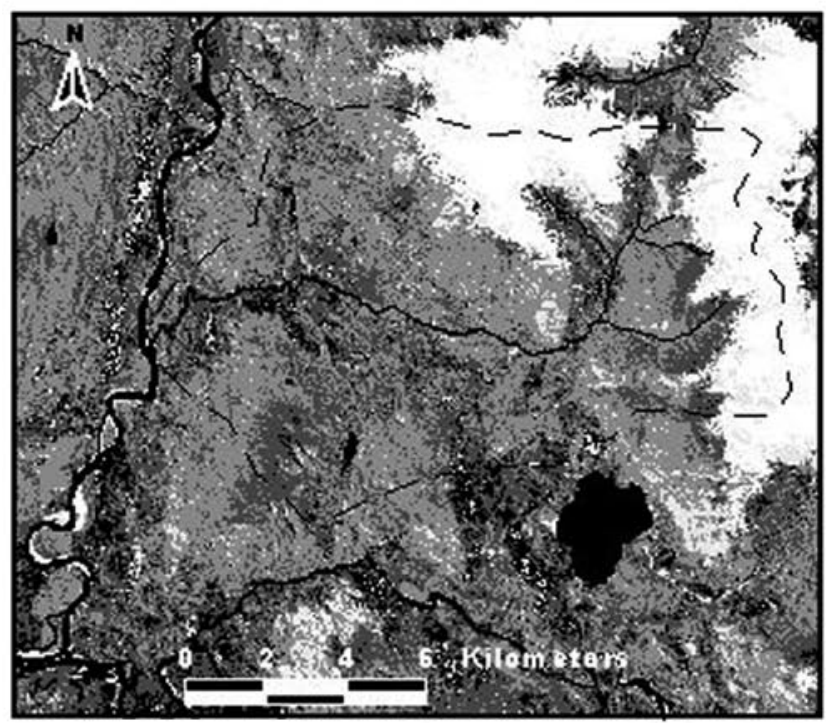

FCV2

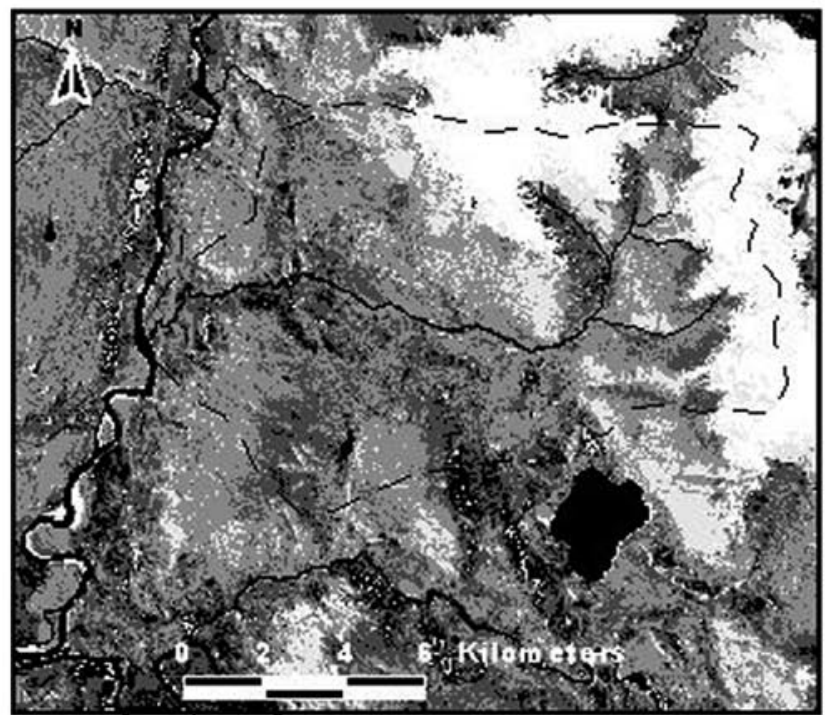

FCV1

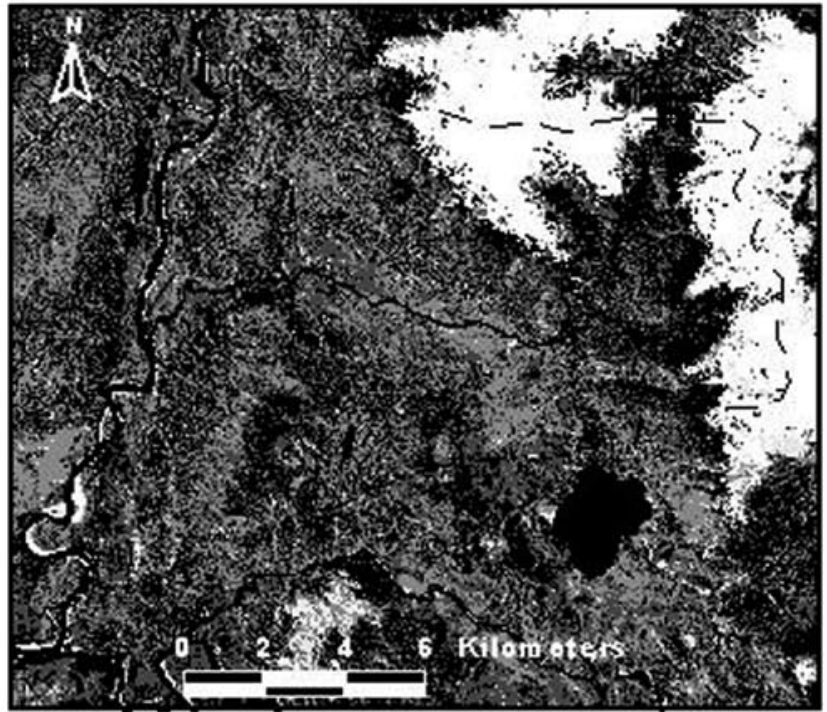

FCV3

Leyenda:

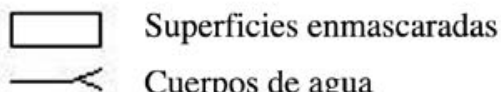

$\because \cdots \quad$ Límite cuenca

Figura 2. Imágenes de FCV de la cuenca del río Mirta y sus alrededores sometidas a distintos niveles de rectificación. FVC0: sin rectificación, FVC1: rectificada atmosféricamente, FCV2: rectificada atmosférica y topográficamente, FCV3: Sometida a rectificaciones atmosférica, topográfica y de subpíxel.

VCF images of the Mirta river basin and its surroundings subjected to different correction levels. VCF0: uncorrected, VCF1: atmospherically corrected, VCF2: atmospherically and topographically corrected, VCF3: subjected to atmospheric, topographic and sub-pixel corrections. 
Cuadro 4. Frecuencia de clases según imagen de NDVI. Frequency classes according to NDVI image.

\begin{tabular}{ccrcrr}
\hline \multirow{2}{*}{ Clase } & \multirow{2}{*}{$\begin{array}{c}\text { Rango de } \\
\text { valores }(\%)\end{array}$} & \multicolumn{2}{c}{ NDVI0 } & \multicolumn{2}{c}{ NDVI1 } \\
\cline { 3 - 6 } & 0 a 25 & 6 & 1.801 & 1 & 316 \\
1 & 25 a 50 & 59 & 17.359 & 2 & 482 \\
2 & 50 a 75 & 35 & 10.157 & 31 & 9.102 \\
3 & 75 a 100 & - & - & 66 & 19.417 \\
4 & - & 100 & 29.317 & 100 & 29.317 \\
\hline
\end{tabular}

Al comparar las clases de ambas imágenes de NDVI, se observa una muy alta concordancia entre las clases 1 $(\mathrm{KIA}=0,95)($ ello se debe a que los píxeles que presentan la clase 1 en NDVI0 aún se mantienen en NDVI1), mientras que la comparación entre las clases 2 y entre las clases 3 no arroja concordancia $(\mathrm{KIA}=0)$.

La figura 3 permite apreciar las diferencias espaciales en la frecuencia de clases de ambas imágenes de NDVI, contribuyendo a explicar los bajos KIAs obtenidos entre sus clases. Destaca particularmente la alta preponderancia de la clase 4 en la imagen de NDVI1, la que ocupa tanto el fondo de valle como diversos sectores de laderas. En NDVI0, la clase 2 se distribuye preferentemente en laderas expuestas al sol y la clase 2 en laderas ocultas al sol. En ambas imágenes, la clase 1 posee una localización similar, que corresponde básicamente a superficies situadas sobre el límite vegetacional.

Relación de la VCF y el NDVI con las coberturas vegetales. El cuadro 5 muestra los valores promedio de VCF y NDVI presentes en cada clase de cobertura vegetal de la cuenca del río Mirta. En términos generales, los mayores valores de VCF y NDVI se observan en las formaciones de bosque achaparrado denso (clase 2), lo que connota que se trata de la clase con mayor superficie ocupada por vegetación en la cuenca, exhibiendo un alto índice de verdor. Los menores valores en tanto, se observan en las formaciones de bosque siempreverde y renoval semidenso (clase 3), seguido de las formaciones de matorral (clases 6 y 7), lo que es atribuible a la menor presencia de biomasa que presentan estas clases en relación con clases de bosque más densas.

Las clases de bosque natural achaparrado corresponden básicamente al tipo forestal lenga (Nothofagus pumilio (Poepp. et Endl.) Krasser), que se localiza aproximadamente entre los 700 y 1.200 m de elevación, a lo largo de laderas con pendientes generalmente pronunciadas. En su límite inferior estas clases colindan con el bosque siem-

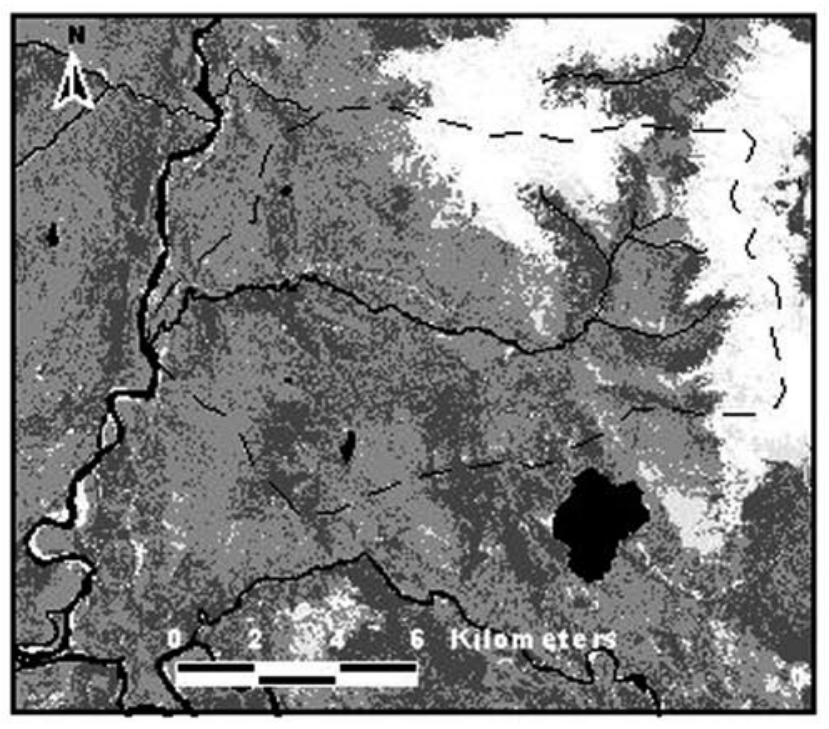

NDVI0

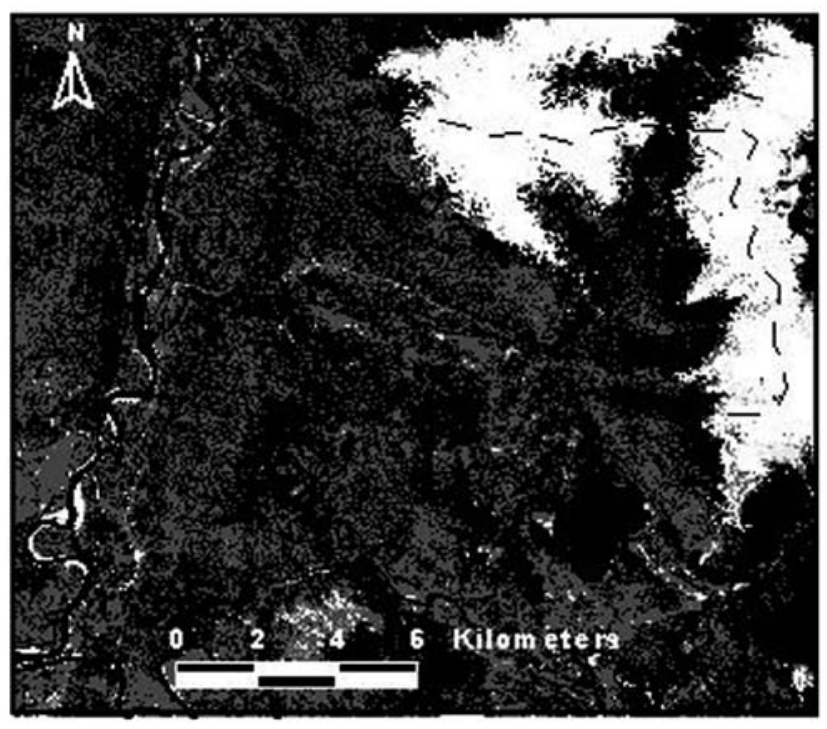

NDVI1
Clases de FCV:

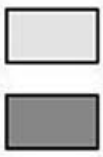

$$
\begin{aligned}
& 1(x<25 \%) \\
& 2(25 \leq x<50 \%)
\end{aligned}
$$

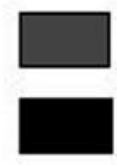

$3(50 \leq x<75 \%)$

$4(x \geq 75)$
Leyenda:

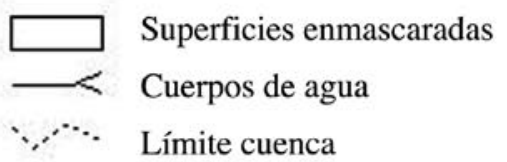

Figura 3. Imágenes de NDVI de la cuenca del río Mirta y sus alrededores. NDVI0 fue estimado sobre una imagen sin rectificación atmosférica y NDVI1 fue estimado sobre una imagen rectificada atmosféricamente.

NDVI images of the Mirta river basin and its surroundings. NDVI0 was estimated on an atmospherically uncorrected image and NDVI1 was estimated on an atmospherically corrected image. 
Cuadro 5. Valores promedio de VCF y NDVI según clase de cobertura vegetal.

VCF and NDVI mean values according to vegetation cover class.

\begin{tabular}{clcc}
\hline \multirow{2}{*}{$\begin{array}{c}\text { Número } \\
\text { de clase }\end{array}$} & Clase de cobertura vegetal & \multicolumn{2}{c}{ Valor promedio (\%) } \\
& & VCF & NDVI \\
\hline 1 & $\begin{array}{l}\text { Bosque natural achaparrado } \\
\text { semidenso }\end{array}$ & 68,14 & 70,39 \\
& $\begin{array}{l}\text { Bosque natural achaparrado } \\
\text { denso }\end{array}$ & 83,29 & 80,90 \\
& $\begin{array}{l}\text { Bosque siempreverde adulto- } \\
\text { renoval semidenso }\end{array}$ & 51,61 & 76,34 \\
& $\begin{array}{l}\text { Bosque siempreverde adulto } \\
4\end{array}$ & 66,48 & 77,82 \\
5 & $\begin{array}{l}\text { semidenso } \\
\text { denso }\end{array}$ & 71,09 & 78,26 \\
6 & Matorral abierto & 61,48 & 75,57 \\
7 & Matorral semidenso & 64,72 & 75,14 \\
\hline
\end{tabular}

preverde y muchas veces coinciden con el límite superior de la especie coihue ( $N$. dombeyi (Mirb.) Oerst.), mientras que en su límite superior colindan con la vegetación de altura distribuida en torno al límite vegetacional. Dentro del bosque achaparrado los valores de VCF y NDVI son apreciablemente más altos en la clase densa que en la clase semidensa.

En cuanto a las clases de bosque siempreverde, es posible apreciar que los mayores valores de VCF y NDVI se localizan en formaciones densas (clase 5), en tanto que los menores valores de ambos parámetros son observados en formaciones semidensas mezcladas con renovales. Las principales especies que constituyen el bosque siempreverde de la cuenca son coihue y tepa (Laureliopsis philippiana (Looser) Schodde), y en menor proporción mañío hembra (Saxegothaea conspicua Lindl.), tineo (Weinmannia trichosperma Cav.) y luma (Luma apiculata (DC.) Burret). Las especies del sotobosque son chilco (Fucsia magellanica Lam.), quila (Chusquea quila Kunth) y colihue (C. culeou E. Desv.). En términos de estructura, este bosque está compuesto por un estrato emergente de coihue, el dosel dominante y codominante está representado por tepa y mañío hembra; el estrato intermedio está compuesto por regeneración avanzada tanto de tepa y mañío, y el estrato sumergido está compuesto por regeneración en estado brinzal de tepa, mañío hembra y luma. En algunos sectores se aprecian las especies tolerantes tepa y mañío hembra, mezcladas con individuos aislados de coihue emergentes. En cuanto a su distribución espacial, el bosque siempreverde ocupa gran parte de las laderas de la cuenca, siendo aquel de mayor densidad el que alcanza las mayores cotas de altitud, colindando en su límite superior con el tipo forestal lenga. No obstante, cuando se mezcla con renovales su localización se aprecia en el fondo de valle.
Las clases de matorral se caracterizan por la presencia de quila, maqui (Aristotelia chilensis (Mol.) Stuntz.) y chilco, cubriendo terrenos bajos, de pendiente suave y bien drenados. Entre las formaciones de matorral abierto (clase 6) y semidenso (clase 7) se aprecian valores similares de NDVI, sin embargo, la VCF es ligeramente superior en esta última.

\section{DISCUSIÓN Y CONCLUSIONES}

El incremento en el nivel de precisión que puede experimentar la estimación de la VCF y el NDVI, una vez aplicadas las correcciones atmosférica, topográfica y de subpíxel, varía de un trabajo a otro, pues cada imagen está sujeta a características únicas como: la hora y la estación del año en que los datos fueron adquiridos, las condiciones atmosféricas y la posición solar al momento de paso del satélite o avión, el ángulo de visión y la anchura de las bandas del sensor, la pendiente y exposición del relieve, el tipo de vegetación imperante en la escena y su condición fenológica y fisiológica. Por otra parte, el modelo de corrección aplicado a la imagen también varía los resultados obtenidos en función de los parámetros de entrada que éste emplee. Al respecto, Riaño et al. (2003) identificaron importantes diferencias en la desviación estándar de diferentes clases de cobertura vegetal al comparar los datos derivados de una imagen topográficamente corregida con un método basado en la división de bandas y otro basado en un DEM. Adicionalmente, aquellos modelos de corrección atmosférica basados en datos in situ (i.e. modelo directo) suelen ser más precisos que aquellos basados en datos tomados del archivo de cabecera de la imagen (i.e. modelo indirecto) (Aronoff 2005).

De acuerdo a Lillesand et al. (2004) y Aronoff (2005), en este trabajo los procedimientos de corrección atmosférica y topográfica producen los resultados esperados sobre los valores de VCF del área de estudio. En el primer caso, el promedio de VCF experimenta una ligera disminución atribuible a la reducción de la radiancia añadida de los píxeles, que atenúa el comportamiento espectral de las coberturas vegetales, incidiendo en la detección de valores de VCF más bajos por parte del algoritmo de descomposición espectral. En el segundo caso, el promedio de VCF experimenta un ligero aumento atribuible a la normalización de la iluminación sobre las coberturas vegetales, incidiendo en la detección de una mayor cantidad de superficies vegetadas por parte del algoritmo de descomposición espectral.

Una vez que es adicionada la corrección de sombras a nivel de subpíxel, la VCF aumenta notablemente su promedio y sus valores se hacen más homogéneos. Destaca particularmente el aumento de la clase de mayores porcentajes de VCF. Además, esta corrección modifica la distribución espacial de los valores de VCF, de tal forma que los mayores porcentajes se observan predominante- 
mente en las laderas ocupadas por bosques densos. Ello difiere de la distribución espacial de los valores de VCF estimados desde la imagen cruda, en donde la exposición del relieve determina la presencia de vegetación y las laderas exhiben valores similares al fondo de valle. Estos resultados enfatizan la importancia de aplicar este tipo de correcciones para calcular parámetros vegetacionales en bosques afectados por un ángulo de elevación solar oblicuo, altas pendientes y sombras generadas por la superficie del dosel vegetal superior.

En la estimación del NDVI la corrección atmosférica produce un aumento notorio de valores, lo cual es atribuible al mayor contraste que se produce entre las bandas rojo e infrarrojo cercano de la imagen al ser reducida la radiancia añadida sobre sus píxeles. En efecto, al examinar la fórmula del NDVI es posible constatar que al producirse una disminución en los valores de ambas bandas el índice resultante aumenta.

Aunque el NDVI minimiza el efecto topográfico, en bosques de coníferas Ekstrand (1996) estimó errores de $7 \%$ en imágenes adquiridas con un ángulo de elevación solar de $38^{\circ}$ y con ángulos de incidencia de entre $37^{\circ}$ y $78^{\circ}$, mientras que Smith et al. (1980) encontraron errores de alrededor de $4 \%$ en imágenes adquiridas con un ángulo de elevación solar de $53^{\circ}$ y con ángulos de incidencia similares. Leprieur y Durand (1988) en tanto, estimaron errores de entre 5\% y $10 \%$ en imágenes adquiridas con un ángulo de elevación solar de $50^{\circ}$ y con ángulos de incidencia de entre $15^{\circ}$ y $60^{\circ}$. Estos resultados permiten deducir que el NDVI atmosféricamente corregido del área de estudio debería contener un porcentaje de error asociado a la permanencia de contrastes en la iluminación de la imagen.

En cuanto al efecto atmosférico sobre la estimación del NDVI, en bosques de Pohutukawa (Metrosideros excelsa Soland. ex Gaertn.) Trotter y Brown (1999) encontraron que el efecto de niebla produjo un error máximo de $18 \%$ en este parámetro, que fue calculado a partir de fotografías multiespectrales adquiridas en diciembre, a las 3:15 p.m hora local, y sometidas a un ángulo de elevación solar de $60^{\circ}$, un azimut solar de $290^{\circ}$ y con un terreno de pendientes inferiores a $15 \%$. Una vez corregido este efecto, el error disminuyó a la mitad y los fuertes contrastes de NDVI se redujeron significativamente. Este resultado remarca las grandes diferencias que pueden existir entre el NDVI crudo y aquel atmosféricamente corregido, lo cual es consistente con los resultados obtenidos en este trabajo.

Por otra parte, al igual que en el caso de la VCF, una vez corregida la imagen, la distribución espacial de los valores de NDVI difiere de aquellos estimados desde la imagen cruda (a excepción de los valores presentes en el límite vegetacional), aumentando fuertemente las superficies cercanas a la saturación de verdor en sectores de laderas ocupadas en gran parte por bosques densos.
Las rectificaciones aquí empleadas adquieren principal relevancia en el modelamiento biofísico de una variable, esto es, en la relación cuantitativa de un parámetro remotamente observado con mediciones in situ (Lillesand et al. 2004). Dado que los efectos atmosférico, topográfico y de subpíxel alteran el valor calculado de VCF y NDVI, sólo es posible asociar correctamente la unidad proporcional obtenida desde la imagen con una unidad de medida física medida en terreno, si la imagen ha sido sometida previamente a las correcciones necesarias. Este hecho ha sido consignado por Brown (2001), quien en un ecotono alpino encontró que los datos de índice de área foliar obtenido en terreno se correlacionaron más fuertemente $(P<0,05)$ con el NDVI atmosféricamente rectificado $(\mathrm{r}=0,43)$ que con aquel sin rectificar $(r=0,16)$.

Aunque este trabajo no relacionó ambos parámetros vegetacionales con mediciones equivalentes in situ, sí pudo constatar a través del empleo de información auxiliar que las formaciones de bosque presentan valores de VCF y NDVI más altos que aquellas de matorral. Además, pudo corroborar que dentro de una misma formación vegetal, tanto la VCF como el NDVI exhiben valores más altos en aquellas clases más densas. Estos resultados son consistentes con la relación directamente proporcional que existe entre ambos parámetros y las medidas de biomasa y abundancia vegetal (Purevdorj et al. 1998, Lillesand et al. 2004, Zheng et al. 2004, Eastman 2006).

Este trabajo demuestra las variaciones apreciables que producen las aplicaciones de procedimientos de corrección de una imagen satelital en la estimación de parámetros vegetacionales de píxel completo (i.e. NDVI) y de subpíxel (i.e. VCF), en especial en bosques afectados por sombras relacionadas a la topografía y a la superficie del dosel vegetal superior, como ocurre en la cuenca del río Mirta. La aplicación de este tipo de procedimientos es de principal importancia si se pretende contribuir a caracterizar y monitorear el recurso bosque mediante el uso de imágenes satelitales, por cuanto facultan la derivación de información más fidedigna en relación con la realidad observada en terreno. El próximo paso implica validar la precisión de los parámetros aquí calculados, para lo cual deberían ser contrastados con mediciones equivalentes obtenidas en terreno, empleando matrices de error y relaciones de asociatividad (e.g. coeficientes de correlación) y concordancia (e.g. KIA).

\section{REFERENCIAS}

Aronoff S. 2005. Remote sensing for GIS managers. New York, USA. ESRI Press. 487 p.

Asner G, J Hicke, D Lobell. 2003. Vegetation indices, spectral mixture and canopy reflectance modeling. In Wulder MA, SE Franklin eds. Remote sensing of forest environment: concepts and case studies. Dordrecht, Holland. Kluwer. p. 209-254. 
Brown DG. 2001. A spectral unmixing approach to leaf area index (LAI) estimation at the alpine treeline ecotone. In Millington AC, Walsh SJ, Osborne PE, Eds. GIS and Remote Sensing Applications in Biogeography and Ecology. Dordrecht, Holland. Kluwer. p. 7-21.

CONAF (Corporación Nacional Forestal, CL), CONAMA (Comisión Nacional del Medio Ambiente, CL), BIRF (Banco Internacional de Reconstrucción y Fomento). 1999. Catastro y evaluación de los recursos vegetacionales nativos de Chile, Informe regional Undécima Región. Santiago, Chile. CONAF. 89 p.

CONAF (Corporación Nacional Forestal, CL). 2000. Proyecto conservación y manejo sustentable del bosque nativo, Área de desarrollo La Junta, Planes de ordenación forestal. Coyhaique, Chile. CONAF. 15 p.

Donoso C. 1981. Ecología forestal, el bosque y su medio ambiente. Santiago, Chile. Editorial Universitaria. 368 p.

Eastman R. 2006. Idrisi Andes guide to GIS and image processing. Worcester, USA. Clark University. 328 p.

Ekstrand S. 1996. Landsat TM-based forest damage assessment: correction for topographic effects. Photogrammetric Engineering \& Remote Sensing 62: 151-161.

Freitas S, M Mello, C Cruz. 2005. Relationships between forest structure and vegetation indices in Atlantic Rainforest. Forest Ecology and Management. 218: 353-362.

Iverson L, RL Graham, EA Cook. 1989. Applications of satellite remote sensing to forested ecosystems. Landscape of Ecology 3(2): 131-143.

Labrecque S, RA Fournier, JE Luther, D Piercey. 2006. A comparison of four methods to map biomass from Landsat-TM and inventory data in western Newfoundland. Forest Ecology and Management. 226: 129-144.

Leprieur CE, JM Durand. 1988. Influence of topography on forest reflectance using Landsat Thematic Mapper and digital terrain data. Photogrammetric Engineering and Remote Sensing 54(4): 491-496.

Lillesand TM, RW Kiefer, JW Chipman. 2004. Remote sensing and image interpretation. New York, USA. Wiley. $763 \mathrm{p}$.

Riaño D, E Chuvieco, J Salas, I Aguado. 2003. Assessment of different topographic corrections in Landsat-TM data for mapping vegetation types. IEEE Transactions on Geoscience and Remote Sensing 41(5): 1056-1061.

Pontius R. 2000. Quantification error versus location error in comparison of categorical maps. Photogrammetric Engineering \& Remote Sensing 66(8): 1011-1016.

Purevdorj Ts, R Tateishi, T Ishiyama, Y Honda. 1998. Relationships between percent vegetation cover and vegetation indices. International Journal of Remote Sensing 19(18): 3519-3535.

Smith JA, TL Lin, KJ Ransom. 1980. The Lambertian assumption and Landsat data. Photogrammetric Engineering and Remote Sensing 46(9): 1183-1189.

Trotter CM, LJ Brown. 1999. Monitoring forest canopy condition by remote sensing. Wellington, New Zealand. Department of Conservation. $19 \mathrm{p}$.

Zheng D, J Rademacher, J Chen, T Crow, M Bresee, J Le Moine, SR Ryu. 2004. Estimating above ground biomass using Landsat 7 ETM+ data across a managed landscape in nothern Wisconsin, USA. Remote Sensing of Environment 93: 402-411. 